\title{
Characterization of exoplanet atmospheres using future space-based infrared telescopes: challenges in detecting biomarkers
}

\author{
Keigo Enya \\ Institute of Space and Astronautical Science, Japan Aerospace Exploration Agency \\ Yoshinodai 3-1-1, Sagamihara, Kanagawa, Japan \\ email: enya@ir.isas.jaxa.jp
}

\begin{abstract}
Characterization of exoplanet atmospheres with space-based infrared telescopes is important to detect biomarkers. A promising method is temporary differential observation. For this method, designs of a wideband infrared spectral disperser are presented. A design using a CdTe prism simultaneously covers $\lambda=1-30 \mu \mathrm{m}$. Designing binary pupil masks for segmented pupils to be used in spatially resolved observations are also shown for another observational method.
\end{abstract}

Keywords. exoplanet, biomarker, infrared, spectral disperser, coronagraph

There are interesting atmospheric absorption features of molecules in the infrared wavelength region, e.g., $\mathrm{H}_{2} \mathrm{O}(1.13,1.38,1.9,2.69,6.2 \mu \mathrm{m}), \mathrm{CO}_{2}(1.21,1.57,1.6,2.03$, $4.25 \mu \mathrm{m}), \mathrm{O}_{3}(4.7,9.1,9.6 \mu \mathrm{m}), \mathrm{O}_{2}(1.27 \mu \mathrm{m}), \mathrm{CH}_{4}(1.65,2.2,2.31,2.37,3.3,6.5,7.7 \mu \mathrm{m})$ and $\mathrm{NH}_{3}(1.5,2.0,2.25,2.9,3.0,6.1,10.5 \mu \mathrm{m})$. Space-based infrared telescopes are useful for observing these features on account of their wavelength coverage, stability and sensitivity. The huge contrast between exoplanets and their parent stars is a serious problem.

\section{Wideband infrared spectrometer}

Precise temporary differential observation is a promising method for the characterization of exoplanet atmospheres, for which wideband infrared spectral dispersers are useful (Enya et al. 2011, Enya 2012, Doyon et al. 2012). Fig. 1 shows new designs of straight-path type dispersers that can be placed into a slot of a mechanical filter wheel in the camera optics. The main prism separates the orders of the spectral format, the grating pattern on the prism surface produces spectral dispersion in each order, and the sub-prism compensates for the inflection angle. The design using $\mathrm{ZnS}$ and $\mathrm{CdTe}$ provides simultaneous wavelength coverage of $1-14 \mu \mathrm{m}$ and $1-30 \mu \mathrm{m}$, respectively. A long observation span, by one or more satellites, is important to improve the signal to noise ratio.

\section{Binary pupil mask coronagraph}

Large telescopes are required for spatially resolved characterization of exoplanets in the infrared, in which telescopes tend to have a segmented pupil. Recent progress in binary pupil mask coronagraphs provides a simple solution for the segmented pupil of a large telescope (Enya \& Abe 2010, Enya et al. 2011, Carlotti, Vanderbei \& Kasdin 2011). Fig. 2 shows designs of binary pupil masks for various segmented pupils. It should be noted that the LOQO optimizer (Vanderbei 1999) was used in the software system for these designs. A very small $I W A, 1.3 \lambda / D$, is also achievable. With this value, it is 

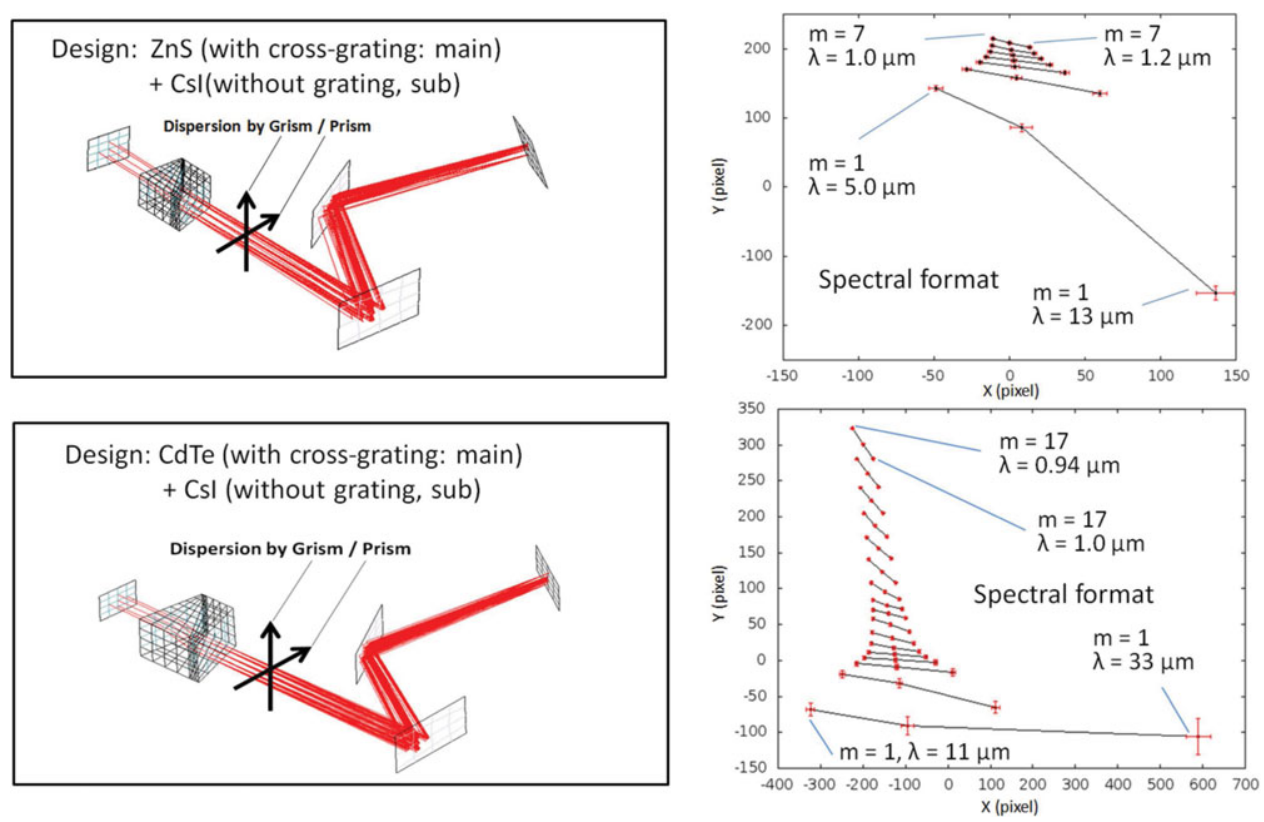

Figure 1. Designs of straight-path spectral dispersers.
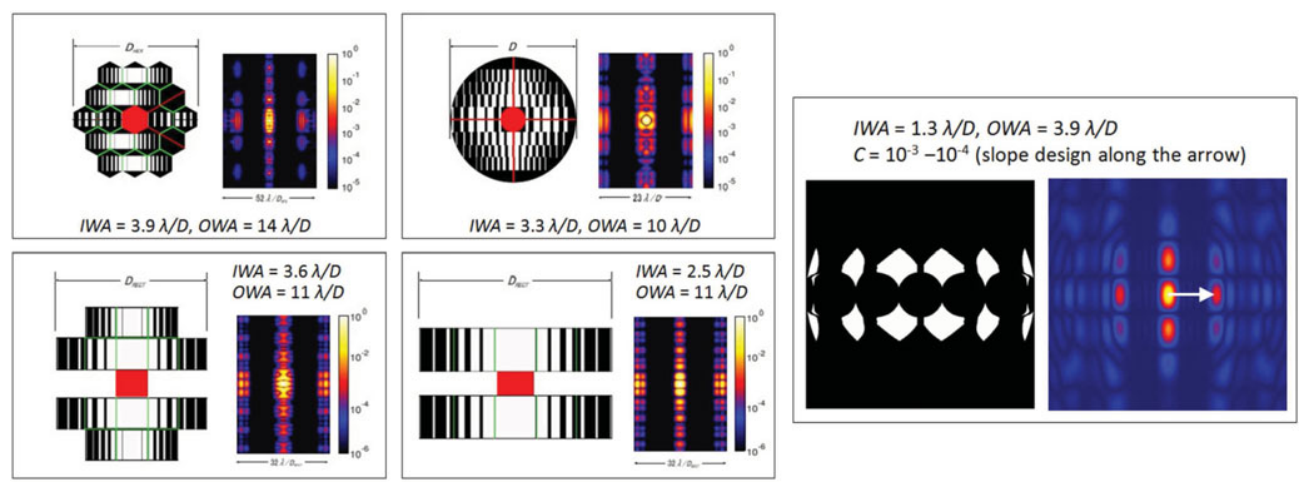

Figure 2. Designs of binary pupil masks for coronagraphs for a segmented telescope pupil.

possible to resolve a small number of stars and their exoplanets close to (or on) the outer edge of the habitable zone (e.g., Kopparapu et al. 2013) at the observing wavelength of $\mathrm{O}_{3}$ even with a $3 \mathrm{~m}$ class telescope. To enhance the contrast, it is necessary to both improve masks and combine other techniques (e.g., PSF subtraction, wavefront correction, and hybridization with a Lyot stop). Ultra-deep observations for very nearby and/or early type stars, including $\alpha$ Cen, Sirius, Procyon, Altair, Vega, and Fomalhaut, are important.

\section{References}

Carlotti, A., Vanderbei, R., \& Kasdin, N. J. 2011 arXiv:1108.4050

Doyon, R. et al. 2012, Proc. SPIE, 8442, 84422R

Enya, K. \& Abe, L. 2010, PASJ, 62, 1407

Enya, K., Abe, L., Takeuchi, S., Kotani, T., \& Yamamuro, T. 2011, Proc. SPIE, 8146, 81460Q

Enya, K. 2012, Proc. SPIE, 8442, 84423Y

Kopparapu, R. K., et al. 2013 ApJ, 765, 131

Vanderbei, R. J. 1999, Optimization methods and software, 11, 451 